\title{
Molecular Identification of Korean Mountain Ginseng Using an Amplification Refractory Mutation System (ARMS)
}

\author{
Jun-Gyo In, Min-Kyeoung Kim, Ok Ran Lee, Yu-Jin Kim, Beom-Soo Lee, \\ Se-Young Kim, Woo-Seang Kwon and Deok-Chun Yang* \\ Korean Ginseng Center and Ginseng Genetic Resource Bank, Kyung Hee University, \\ Seocheon, Giheung-gu Yongin-si, Gyeonggi-do 449-701, South Korea \\ (Received January 11, 2010; Revised February 10, 2010; Accepted February 12, 2010)
}

\begin{abstract}
Expensive herbs such as ginseng are always a possible target for fraudulent labeling. New mountain ginseng strains have occasionally been found deep within mountain areas and commercially traded at exorbitant prices. However, until now, no scientific basis has existed to distinguish such ginseng from commonly cultivated ginseng species other than by virtue of being found within deep mountain areas. Polymerase chain reaction (PCR) analysis of the internal transcribed spacer has been shown to be an appropriate method for the identification of the most popular species (Panax ginseng) in the Panax ginseng genus. A single nucleotide polymorphism (SNP) has been identified between three newly found mountain ginseng (KGD4, KGD5, and KW1) and already established Panax species. Specific PCR primers were designed from this SNP site within the sequence data and used to detect the mountain ginseng strains via multiplex PCR. The established multiplex-PCR method for the simultaneous detection of newly found mountain ginseng strains, Korean ginseng, and foreign ginseng in a single reaction was determined to be effective. This study is the first report of scientific discrimination of "mountain ginsengs" and describes an effective method of identification for fraud prevention and for uncovering the possible presence of other, cheaper ginseng species on the market.
\end{abstract}

Key words : amplification refractory mutation system (ARMS)-PCR, multiplex PCR, Panax ginseng, single nucleotide polymorphism (SNP)

\section{INTRODUCTION}

Ginseng (Panax ginseng) is one of the most important medicinal plants in East Asia, throughout which almost every species of the genus has been employed as a source of medicine. Ginseng literally means "the essence of the human" [1] and is also known as the "king of herbs." China and Korea have utilized this plant for more than 2000 years as a tonic, a stimulant, and an agent to foster fatigue and stress-resistance [2]. Recently, the pharmaceutical effects of ginseng roots have been demonstrated by a host of studies and ginseng has become a world-renowned medicinal plant. The active constituents contained in most ginseng species include ginsenosides, polysaccharides, peptides, polyacetylenic alcohols, and fatty acids [3]. Ginseng is known to improve antibody-dependent cell cytotoxicity [4], reduce lung pathology [5], bolster learning in mice [6], potentiate vaccination against the common cold

\footnotetext{
* Corresponding author. E-mail: dcyang@khu.ac.kr
} Phone: +82-31-201-2100, Fax: +82-31-201-2687 and/or influenza [7], inhibit the development of reverse tolerance to morphine [8], prevent injuries from oxygen free radicals [9], exert anti-stress effects [10], inhibit mutagenesis [11], potentiate the generation of nerve fiber [12], and exhibit antiaging properties [13].

Ginseng belongs to the genus Panax, which consists of 17 species, only some of which have been widely cultivated due to their profound medicinal effects. These are $P$. ginseng (Korean ginseng), P. japonicus (Japanese ginseng), P. notoginseng (Chinese ginseng), and P. quinquefolius (American ginseng) [10]. Normally, cultivated ginseng is systematically farmed on open land with proper control of sunlight by shielding (canopy) that helps to reduce 1/8$1 / 13$ of total sunlight and is harvested after a 5-6 year cultivation period. Occasionally, however, indigenous mountain ginseng has been discovered and traded at very high prices. Mountain ginseng, found deep within mountain areas at altitudes between 800 and $1,500 \mathrm{~m}$, is much slower in growth and more sensitive to environmental changes than cultivated varieties and exhibits a preference 
for areas with fluctuating daily temperatures and minimal exposure to direct sunlight. The hardiness of mountain ginseng has been commonly thought to result in more pharmaceutically active roots and some mysterious effects on the human body.

Traditionally, authentication of ginseng has relied upon morphological and histological inspections, but in many cases, such approaches are far from reliable. For example, most ginseng roots are extremely morphologically similar, thus rendering their differentiation almost impossible for the layperson. The illegal practice of disguising common cheap ginseng as mountain ginseng has become a more prevalent problem in recent years. Determining the source of a plant from its morphology is frequently difficult, as the majority of ginseng products are sold in the form of powder or shredded slices.

In eukaryotes, two internal transcribed spacers (ITS1 and ITS2) that flank the 5.8S rDNA region have been identified, which are co-transcribed with the 5.8S rDNA, but not translated [14]. The ITS sequences are highly conserved throughout plant species but are suitable markers for the interspecies variation and intraspecies conservation. Thus, ITS sequences are suitable targets for investigations of plant phylogenetic relationships within the same genus and species [15-18]. The phylogenetic relationship of the Panax genus has been more recently studies and so the relationship of any new strains found within Panax could be better positioned.

In this study, we found three new mountain ginseng strains through the analysis of the ITS regions. The ITS sequences were different from those of already established Korean ginseng and other foreign ginseng. This report presents a more reproducible and robust approach for the authentication and differentiation of a mountain ginseng from already established ginseng species via single nucleotide polymorphism (SNP) analysis.

\section{MATERIALS AND METHODS}

\section{Ginseng materials}

Actively growing ginseng roots were collected from Korean Ginseng Center and Ginseng Genetic Resource Bank, Kyung Hee University, Republic of Korea. Five samples of ginseng were selected : Panax ginseng JaKyung variety, $P$. ginseng Chun-Poong cultivar, $P$. japonicus, $P$. notoginseng, and $P$. quinquefolius. Three samples of mountain ginseng roots were obtained from Odaesan (Mt. Odae) by "mountain ginseng diggers," also known as simmani in the Korean language.

\section{DNA extraction and PCR of ITS region}

DNA from the ground samples was isolated and purified using a plant-DNA isolation kit (NucleoSpin Plant; Macherey-Nagel, Düren, Germany). The oligonucleotide primers for the amplification of the ITS region were synthesized by Genotec, Inc. (Daejeon, Korea). The universal primers that annealed at the 5'- and 3'-ends of the ITS region were ITS5F (5'-GGAAGTAAAAGTCGTAACAAGG3') and ITS4R (5'-TCCTCCGCTTATTGATATGC-3'), respectively [14]. PCR amplification was conducted in a 100-il final reaction volume, and the reaction mixture consisted of $1.0 \mu \mathrm{M}$ of each primer, $50 \mathrm{ng}$ of extracted DNA, $0.2 \mathrm{mM}$ of each deoxynucleoside triphosphate (dNTP), $1.5 \mathrm{mM} \mathrm{MgCl}_{2}$, 10× reaction buffer (Biogen, Daejeon, Republic of Korea), and 1 unit of Taq DNA polymerase (Biogen). PCR was conducted for 30 cycles, with a thermal profile as follows: predenaturation at $94^{\circ} \mathrm{C}$ for 10 minutes, denaturation at $94^{\circ} \mathrm{C}$ for 30 seconds, primer annealing at $68^{\circ} \mathrm{C}$ for 30 seconds, and extension at $72^{\circ} \mathrm{C}$ for 30 seconds. The final cycle included a 10 minute extension at $72^{\circ} \mathrm{C}$ to ensure full extension of the products.

\section{Gel electrophoresis and DNA sequencing}

The PCR products were analyzed via electrophoresis of a $5-\mu \mathrm{l}$ aliquot through a $1.0 \%$ (wt/vol) agarose gel (Caledon, Geogetown, ON, Canada) stained with ethidium bromide and visualized via UV transillumination. A 100-bp ladder (Bioneer, Daejeon, Republic of Korea) was employed as a size marker. The DNA from all samples was PCRamplified and purified with a PCR-product purification kit (Suprec-02; Dakara, Korea), in accordance with the manufacturer's instructions. The purified PCR products were sequenced by Genotec (Daejeon, Korea).

\section{ITS sequence comparison}

The DNA sequences obtained in the sequencing experiments were then used to conduct a comparison of the ITS regions. The entire sequence of the ITS1-5.8S-ITS2 was compiled using SeqMan software and the sequences were edited with the BioEdit software program [19]. The ITS sequences of the related Panax species were acquired from GenBank. Multiple alignments were conducted using the CLUSTAL X software program [20].

\section{Design of specific primers}

Two specific primers (quinR and ginR) for analyzing the mountain ginseng samples were designed on the basis of the SNP sites specific to the three mountain ginseng 
samples. The oligonucleotide primers were synthesized and prepared by Genotec. The orientation of the specific primers (quinR and ginR) and common primers (F1 and F2) in the ITS1-5.8S-ITS2 region are shown in Fig. 1. The sequences of the four primers used to detect SNPs are shown in Table 1. The underlined base pair (bp) next to the species-specific 3'-base (in bold) in specific primers quinR and ginR was deliberately designed to ensure absolute specificity via nucleotide substitution. We also developed the new common primers, F1 and F2, for the amplification of shorter DNA bands with specific electrophoresis primers, which facilitated the visualization of easily separated DNA bands.

\section{Multiplex PCR}

Multiplex PCR was conducted with identical concentrations and temperature conditions as described above, except for the following: the concentration of the primer S1 was $0.12 \mu \mathrm{M}$ and the concentrations of the primers S3, $\mathrm{A} 2$, and A3 were $0.2 \mu \mathrm{M}$. PCR was conducted for 30 cycles, with a thermal profile as follows: pre-denaturation at $94^{\circ} \mathrm{C}$ for 10 minutes, denaturation at $94^{\circ} \mathrm{C}$ for $45 \mathrm{sec}-$ onds, primer annealing at $62^{\circ} \mathrm{C}$ for 45 seconds, and extension at $72^{\circ} \mathrm{C}$ for 45 seconds.

\section{RESULTS}

DNA sequencing of ITS1-5.8S-ITS2 region of Panax species and mountain ginseng

The ITS1-5.8S-ITS2 rDNA regions were PCR-amplified from the roots of eight samples from $P$. ginseng JaKyung variety, $P$. ginseng Chung-Poong cultivar, $P$. japonicus, $P$. notoginseng, $P$. quinquefolius, and three mountain ginseng samples using the ITS5F and ITS4R universal primer sets. More than 700 bp of the rDNA region was PCR-amplified containing partial 18S rDNA, complete ITS1, complete 5.8S rDNA, complete ITS2, and partial 26S rDNA (Fig. 1). The ITS1, 5.8S rDNA, and ITS2 sequences were determined to be 222, 163, and $234 \mathrm{bp}$, respectively. The sequences of the Japanese ginseng $(P$. japonicus), Chinese ginseng ( $P$. notoginseng), and American ginseng ( $P$. quinquefolius) were identical to their corresponding NCBI GenBank sequences. The NCBI GenBank accession numbers for the sequences of the ITS1-5.8SITS2 regions of the two $P$. ginseng species are AB043871 (Ja-Kyung variety) and AF274534 (Chung-Kyung cultivar).

\section{Alignment of DNA sequences of the ITS1-5.8S-ITS2 regions}

The DNA sequences of the ITS1-5.8S-ITS2 region of

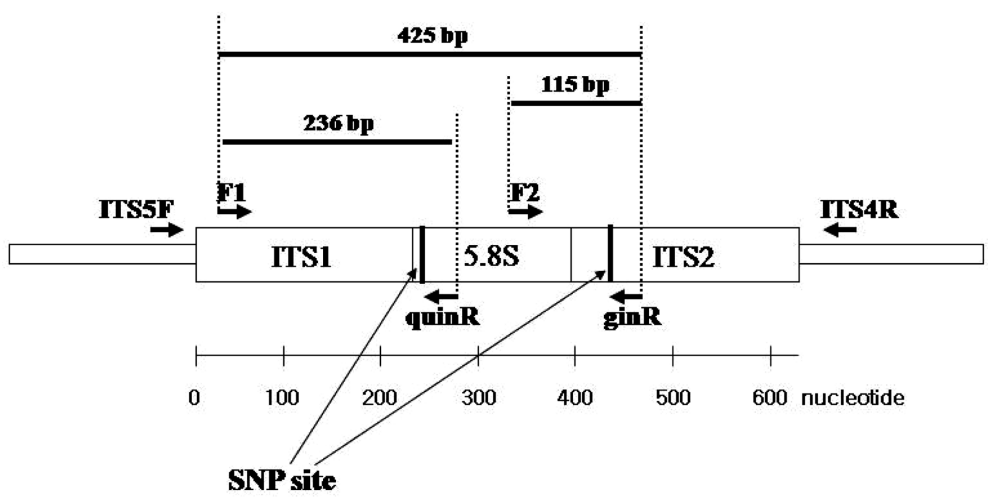

Fig. 1. A diagram of the nuclear ITS region and the positions of the primer sets used for PCR in this study. ITS: Internal transcribed spacer.

Table 1. Oligonucleotide sequences of primers used for PCR

\begin{tabular}{|c|c|c|c|c|c|}
\hline $\begin{array}{l}\text { Primer } \\
\text { name }\end{array}$ & Sequence & Size & Position & $\operatorname{Tm}(\mathrm{C})$ & Specificity \\
\hline F1 & 5' - GAC CCG CGA ACA CGT TAC AAT ACT G - 3' (CT) & 25 mer & $23 \sim 47$ & 61 & common \\
\hline $\mathrm{F} 2$ & 5' - CAA GTT GCG CCC GAA GCC ATT TG - 3' (AT) & 23 mer & $333 \sim 355$ & 60 & common \\
\hline quinR & 5' - TGC GAG AGC CGA GAT ATC CGT TTC - 3' (GT) & 24 mer & $258 \sim 235$ & 62 & $\begin{array}{l}\text { specific for wild ginseng } \\
\text { and } P \text {. quinqufolius }\end{array}$ \\
\hline $\operatorname{gin} R$ & 5' - CCA TTA TCC GCC CCT CCG CCT TA - 3' (CT) & 23 mer & $447 \sim 425$ & 65 & $\begin{array}{l}\text { specific for wild ginseng } \\
\text { and } P \text {. ginseng }\end{array}$ \\
\hline
\end{tabular}


the three mountain ginseng samples were aligned with other Panax species' DNA sequences. In the sequence alignment of the ITS1-5.8S-ITS2 region, the total length of the ITS-5.8S-ITS2 region of the Panax species was determined to be $619 \mathrm{bp}$. We did not detect any additional nucleotides in the region, although we observed some variations including nucleotide deletions, transitions, and transversions.

As expected, the sequences of the two varieties and cultivars of $P$. ginseng exhibited complete homology (100\% identical) in the ITS1-5.8S-ITS2 region (data not shown). Using the alignment results, a host of SNP sites specific to mountain ginseng was detected. Following our objective to develop a new method for the differentiation of mountain ginseng from other Korean ginseng species ( $P$. ginseng) and foreign Panax species, we attempted to delineate a SNP site that was specific for mountain ginseng. Unfortunately, no SNP site specific for mountain ginseng was identified. However, two SNP sites, one of which was specific for the mountain ginseng and foreign (non-Korean) ginseng species ( $P$. japonicus, $P$. notoginseng, $P$. quinquefolius), and the other of which was specific for the mountain ginseng and the Korean ginseng species ( $P$. ginseng), were identified. The first SNP site specific for the mountain ginseng and foreign (nonKorean) ginseng was located at nucleotide position 235 in the ITS1-5.8S-ITS2 region (Fig. 2). The $235^{\text {th }}$ nucleotide in the ITS1-5.8S-ITS2 region was A in all Korean ginseng species, but $\mathrm{G}$ in mountain ginseng and other foreign (non-Korean) Panax species in the NCBI GenBank database. The $425^{\text {th }}$ nucleotide in the ITS1-5.8S-ITS2 region was $\mathrm{T}$ in mountain ginseng and Korean ginseng species, but $\mathrm{C}$ in other foreign (non-Korean) Panax species. In the ITS1-5.8S-ITS2 region, DNA sequences of three mountain ginseng samples were so similar to those of Korean ginseng species $(P$. ginseng) that only one base difference was found in the $235^{\text {th }}$ nucleotide position.

\section{Multiplex ARMS-PCR}

Molecular identifications were conducted via analyses of the ITS regions of Panax species using species-specific ARMS PCR. All experiments were repeated multiple times to confirm the reproducibility of the data. The common forward primers F1 and F2, and two specific primers quinR (a specific primer for mountain ginseng and foreign ginseng) and ginR (a specific primer for mountain ginseng and Korean ginseng) (Table 1), were designed using the available SNP sites of the ITS1-5.8S-ITS2 region of the Panax species to develop an ARMS-PCR mountain ginseng detection method using multiplex PCR. The two common forward primers were determined to be common to all Panax species (whether these pairs are Panax-specific is not currently known). The combination of one specific primer (quinR) with one of the common primers (F1) yielded one amplicon of band size 236, and the combination of the other specific primer (ginR) with the two common primers (F1 and F2) yielded two amplicons of band size 115 and 425 . The relative position of the four primers and the sizes of the expected species-specific amplicons are shown in Fig. 1. When this method was applied to mountain ginseng, Korean ginseng ( $P$. ginseng Ja-Kyung variety and $P$. ginseng Chun-Poong cultivar), Japanese ginseng ( $P$. japonicus), Chinese ginseng ( $P$. notoginseng), and American ginseng ( $P$. quinquefolius), only mountain ginseng produced all the three amplicons, with sizes of $115 \mathrm{bp}, 236 \mathrm{bp}$, and $425 \mathrm{bp}$. The two Korean ginseng samples yielded the expected two amplicons, with sizes of $115 \mathrm{bp}$ and $425 \mathrm{bp}$ (Fig. 3A and B). All three of the foreign Panax species yielded the only expected amplicon with a size of $236 \mathrm{bp}$ (Fig. 3B). Note that this method

\begin{tabular}{|c|c|c|c|}
\hline Panax species & Sequences at mucleotide 235 & Sequences at nucleotide 425 & $\begin{array}{c}\text { Accession } \\
\text { No. }\end{array}$ \\
\hline P. gïrseng & 250 & 440 & \\
\hline Ja-Kyung (var.) & CICGACAACGGATATCICGGCICICGCATC & GAGITGAGGCGGAGGGGCGGATAATGGCCT & AF043871 \\
\hline Chun-Poong(cul) & CTOGACAACGGATATCICGGCICTCGCATC & GAGपIGAGGCGGAGGGGCGGATAATGGCCT & AF274533 \\
\hline KGD4 & CTOGGCAACGGATATCTCGGCICTCGCATC & GAGIIGAGGCGGAGGGGCGGATAATGGCCT & \\
\hline KGDS & CTCGGCAACGGATATCTCGGCTCTCGCATC & GAGMIGAGGCGGAGGGGCGGATAATGGCCT & \\
\hline KW1 & CICGGCAACGGATATCICGGCICTCGCATC & GAGLIIAGGCGGAGGGGCGGATAATGGCCT & \\
\hline P.japonices & CTCGQCAACGGATATCTCGGCTCTCGCATC & GAGTCGAGGCGGAGGG-CGGATAATGGCCT & AY233323 \\
\hline P. notogürseng & CIOGGCAACGGATATCIOGGCICTCGCATC & GAGTCGATGCGGAGGGGCGGATAATGGOCT & U41685 \\
\hline P. qǖnquefoläs & CTOGGCAACGGATATCTOGGCTCTCGCATC & GAGTCGAGGCGGAGGGGCGGATAATGGCCT & U41688 \\
\hline
\end{tabular}

Fig. 2. Comparison of the ITS1-5.8S-ITS2 sequences of the mountain ginseng, $P$. ginseng varieties, and cultivars, as well as those of three major foreign ginseng species. 


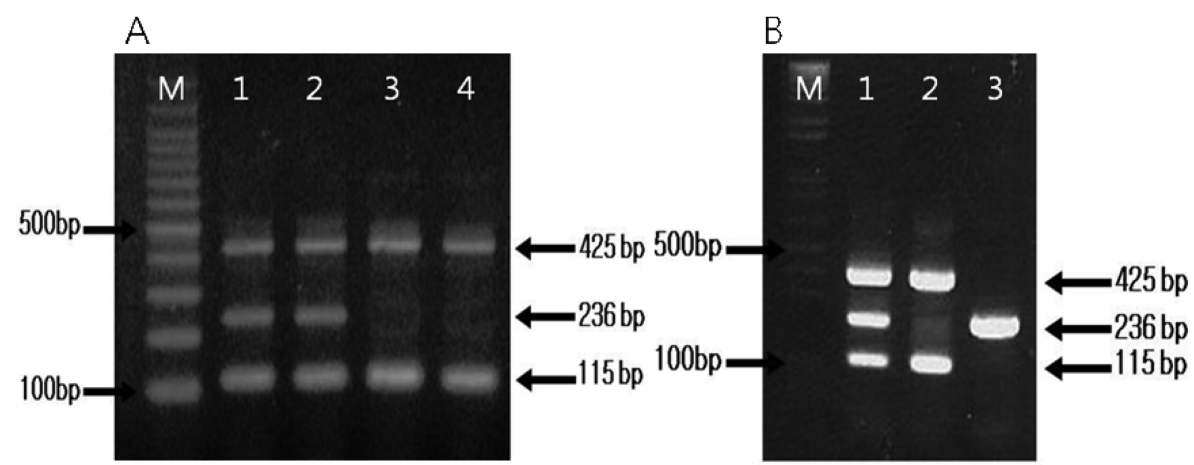

Fig. 3. PCR products in multiplex PCR using the primers, F1, F2, quinR and ginR.

A. Lane M: 100 bp DNA Ladder; lane 1: mountain ginseng KGD4, lane 2: mountain ginseng KW1, lane 3: P. ginseng Ja-Kyung variety, lane 4: $P$. ginseng Chun-Poong cultivar.

B. Lane M: 100 bp DNA Ladder; lane 1: mountain ginseng KGD4, lane 2: P. ginseng Ja-Kyung variety, lane 3: P. quinquefolius (American ginseng).

does not distinguish among the three foreign species of ginseng, and this level of differentiation was beyond the scope of this work. We confidently conclude that ARMSPCR techniques indeed can be used to specifically detect mountain ginseng in vitro among all Panax species.

\section{DISCUSSION}

\section{Morphological description}

Three major ginseng species, $P$. ginseng (Korean ginseng), P. notoginseng (Chinese ginseng), and $P$. quinquefolius (American ginseng), are considered most medicinally relevant on the basis of the quantity of triterpenoids (ginsenosides) and seed morphology [21], and are commonly commercially utilized. In this study, we analyzed the three principal Panax species in addition to Japanese ginseng (P. japonicus). Until now, no standard method had been established to identify newly found mountain ginseng based on morphological analyses. Therefore, an easy and reliable method that can distinguish newly found mountain ginseng was needed that could have multiple, important applications.

\section{Multiplex ARMS-PCR}

In this study, we successfully employed ARMS-PCR techniques to distinguish newly found mountain ginseng strains from other Panax species. This method appears reasonably applicable to more mountain ginseng samples yet to be found. This report is the first to show that newly found mountain ginseng can be distinguished from already established ginseng species. A real possibility of discovering other new mountain ginseng strains remains.
Similar strains that share the same ITS sequences with KGD4, KGD5, and KW1 could be easily determined, but more importantly, the phylogenetic positions of new mountain ginseng strains could be confirmed using ITS sequencing and distinguished using their SNP sites, as shown in this study. Our ARMS-PCR primers could be employed in the analysis and identification of more mountain ginseng samples to determine whether such samples are truly different from cheaper, common varieties. However, problems remain in that if any newly found mountain ginseng varieties have the same ITS sequence with those of previously established species such as $P$. ginseng (Korean ginseng), P. notoginseng (Chinese ginseng), and $P$. quinquefolius (American ginseng), then this method cannot be applied to differentiate between newly found ginseng.

Denaturing gradient gel electrophoresis (DGGE) is another technique that can be used to differentiate newly discovered mountain ginseng from cultivated varieties. However, ARMS-PCR methods tend to require less sophisticated equipment and shorter analysis times than those associated with DGGE. Thus, ARMS-PCR may represent the optimal method for the routine analysis of cultures in the laboratory.

In conclusion, the authentication of newly found mountain ginseng has traditionally relied upon morphological inspections. However, no scientific basis was established and determining the source of an herb from its morphology is often difficult. Therefore, a method of gene analysis, rather than the traditional authentication methods, is clearly desirable. Among a host of molecular biological methods (e.g., RAPD, RFLP, AFLP), we have described a 
more reproducible and robust approach to the differentiation of newly found mountain ginseng using SNP analysis. Using this method, we have successfully distinguished new mountain ginseng samples from already established ginseng species.

\section{ACKNOWLEDGMENTS}

This study was supported by KGCMVP (Korean Ginseng Center for Most Valuable Products \& Ginseng) for Technology Development Program of Agriculture and Forestry, Ministry for Food, Agriculture, Forestry and Fisheries, Republic of Korea.

\section{REFERENCES}

1. Jorgensen RA, and Cluster PD. Modes and tempos in the evolution of nuclear ribosomal DNA: new characters for evolutionary studies and new markers for genetic and population studies. Annals Miss Bot Garden 75(4): 1238-1247 (1998)

2. Cho JS, Han Y N, Oh HI, Park H, Sung HS, Park JI (Eds.). Understanding of Korean Ginseng: Korean ginseng contains various effective components. The Society for Korean Ginseng, Seoul, Korea, p 39-41 (1995)

3. Lee FC, Elizabeth NJ, (Ed.). Facts about ginseng, the elixir of life. Hollyn International Corp. (1992)

4. See DM, Broumand N, Sahl L, Tilles JG. In vitro effects of Echinacea and ginseng on natural killer and antibody-dependent cell cytotoxicity in healthy subjects and chronic fatique syndrome or acquired immunodeficiency syndrome patients. Immunopharm. 35(3): 229-235 (1997)

5. Song ZJ, Johansen HK, Faber V, Hoiby N. Ginseng treatment reduces bacterial load and lung pathology in chronic Pseudomonas aeruginosa pneumonia in rats. Antimicrob. Agents Chemo. 41(5): 961-964 (1997)

6. Nishiyama N, Chu PJ, Saito H. An herbal prescription, S$113 \mathrm{~m}$, consisting of biota, ginseng and schizandra, improves learning performance in senescence accelerated mouse. Biol Pharm Bull. 19(3): 388-393 (1996)

7. Scaglione F, Cattaneo G, Alessandria M, Cogo R. Efficacy and safety of the standardized ginseng extract $G 115$ for potentiating vaccination against common cold and/or influenza syndrome. Drugs Under Exp Clin Res. 22(2): 65-72 (1996)

8. Kim HS, Kang JG, Oh KW. Inhibition by ginseng total saponin of the development of morphine reverse tolerance and dopamine receptor supersensitivity in mice. General pharm. 26(5): 1071-1076 (1995)
9. Kim HY. Ginsenosides protect pulmonary vascular endothelium against free radical induced injury. Biochem Biophys Res Comm. 189(2): 670-676 (1992)

10. Takahashi M. Anti-stress effect of ginseng on the inhibition of the development of morphine tolerance in stressed mice. Jpn J Pharm. 59(3): 399-404 (1992)

11. Rhee YH. Inhibition of mutagenesis and transformation by root extracts of Panax ginseng in vitro. Planta Medica 57(2): 125-128 (1991)

12. Takemoto Y. Potentiation of nerve growth factor-mediated nerve fiber production in organ cultures of chicken embryonic ganglia by ginseng saponins: Structure-activity relationship. Chem Pharm Bull. (Tokyo) 32(8): 3128-3133 (1984)

13. Chang HM, Yeung HW, Tso WW, Koo A (Eds.). Chemical and biochemical studies on antioxidant components of ginseng. In: Advances in Chinese Medicinal Materials Research. World Scientific Press, Singapore, p 485-498 (1985)

14. Innis MA, Gelfand DH, Sninsky JJ, White TJ. PCR protocols. A guide to methods and applications: Amplification and direct sequencing of fungal ribosomal RNA genes for phylogenetics. Academic Press, San Diego, p 315-322 (1990)

15. Baldwin BG. Phylogenetic utility of the internal transcribed spacers of nuclear ribosomal DNA: an example from the Comositae. Mol. Phylogenet. Evol. 1(1): 3-16 (1992)

16. Guo Y, Kondo K, Terabayashi S, Yamamoto Y, Shimada H, Fujita M, Kawasaki T, Maruyama T, Goda Y, Mizukami H. DNA authentication of So-jutsu (Atractylodes lancea rhizome) and Byaku-jutsu (Atractylodes rhizome) obtained in the market based on the nucleotice sequence of the $18 \mathrm{~S}-5.8 \mathrm{~S}$ rDNA internal transcribed spacer region. J Nat Med. 60: 149-156 (2006)

17. Howard C, Bremner PD, Fower MR, Isodo B, Scott NW, Slater A. Molecular identification of Hypericum perforatum by PCR amplification of the ITS and 5.8S rDNA region. Planta Med. 75: 864-869 (2009)

18. Suh Y, Thien LB, Reeve HE, Zimmer EA. Molecular evolution and phylogenetic implications of internal transcribed spacer sequences of ribosomal DNA variation in Winteraceae. Am J Bot 80(9): 1042-1055 (1993)

19. Hall TA. BioEdit: A user-friendly biological sequence alignment editor and analysis program for Windows 95/98/NT. Nucleic Acids Symp Ser. 41: 95-98. (1999)

20. Thompson JD, Gibson TJ, Plewniak F, Jeanmougin F, Higgins DG. The ClustalX windows interface: flexible strategies for multiple sequence alignment aided by quality analysis tools. Nucleic Acids Res. 25(24): 4876-4882 (1997)

21. Zhou J, Huang WG., Wu MZ, Yang CR, Feng KM, Wu ZY. Triterpenoids from Panax Linn. and their relationship with taxonomy and geographical distribution. Acta Phytotax Sin. 13(2): 29-45 (1975) 